\title{
Endoscopic duodenal mucosal resurfacing for nonalcoholic steatohepatitis (NASH): a pilot study
}

\section{(ㄷ)(1) $\odot$}

\section{Authors}

Alia Hadefi ${ }^{1,2}$, Laurine Verset ${ }^{3}$, Martina Pezzullo ${ }^{4}$, Nicolas Rosewick², Delphine Degré ${ }^{1,2}$, Thierry Gustot ${ }^{1,2}$, Christophe Moreno ${ }^{1,2}$, Jacques Devière ${ }^{1,2}$, Eric Trépo ${ }^{1,2}$

Institutions

1 Department of Gastroenterology,

Hepatopancreatology, and Digestive Oncology, CUB

Hôpital Erasme, Université Libre de Bruxelles, Brussels, Belgium

2 Laboratory of Experimental Gastroenterology, Université Libre de Bruxelles, Brussels, Belgium

3 Department of Pathology, Institut Jules Bordet, Université Libre de Bruxelles (ULB), Brussels, Belgium

4 Department of Radiology. CUB Hôpital Erasme, Université Libre de Bruxelles, Brussels, Belgium

submitted 26.4.2021

accepted after revision 7.6.2021

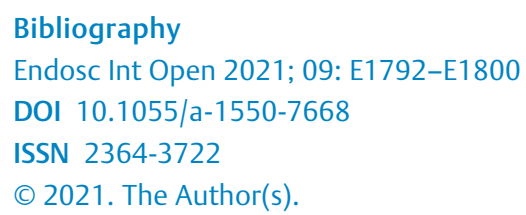

\author{
\# Supplementary material is available under \\ https://doi.org/10.1055/a-1550-7668
}

\section{ABSTRACT}

Background and study aims Nonalcoholic steatohepatitis (NASH) is a leading cause of chronic liver disease worldwide with limited treatment options. Duodenal mucosal resurfacing (DMR) has been associated with improvement in glycaemic parameters and liver function tests (LFTs) in type 2 diabetes. This study aimed to assess the effect of DMR in patients with NASH.

Patients and methods This was a single-center, open-label pilot study. Patients with definite, biopsy-proven NASH (nonalcoholic fatty liver disease activity score [NAS] $\geq 4$ ) underwent a single DMR procedure followed by a 2-week postprocedural diet, without lifestyle intervention. The primary outcome was either resolution of NASH with no worsening of fibrosis or improvement in fibrosis ( $\geq 1$ stage) with no worsening of NASH at 12 months. Secondary outcomes were changes in key histological parameters of NASH, surrogate markers of fibrosis, LFTs, and metabolic factors at 12 months.

Results From 2017 to 2019, 14 patients underwent successful DMR, of whom 11 were included in the analysis. After 12 months, no resolution of NASH was observed, while three patients (27\%) had marginal improvement in fibrosis with no worsening of NASH. Serious adverse events related to the procedure were reported in two patients out of $14(14 \%)$. Neither weight loss nor improvement in NAS score, or in the other secondary outcomes, were observed at 12 months.

Conclusions In this small and heterogenous study population, we found that DMR, in the absence of lifestyle intervention, did not induce NASH resolution and marginally improved liver fibrosis at 12 months.

\section{Introduction}

Nonalcoholic fatty liver disease (NAFLD) affects up to $25 \%$ of the worldwide population [1]. It is the leading cause of chronic liver disease in high-income countries and ranges from isolated steatosis to nonalcoholic steatohepatitis (NASH), which is characterized by hepatocyte injury and inflammation that can evolve to cirrhosis and/or hepatocellular carcinoma [2,3]. The 
main risk factors for NAFLD are obesity and type 2 diabetes mellitus (T2DM) [4]. More specifically, insulin resistance (IR) in peripheral tissues is considered to be one of the cornerstones of $\mathrm{NASH}$, and this can be positively improved by weight loss [5], which is also the only validated treatment for NASH [6-9]. However, only a minority of patients can maintain and sustain longterm weight loss [6]. Despite numerous ongoing pharmaceutical trials, there is still an unmet need for a longstanding effective treatment to tackle NASH.

Duodenal mucosal resurfacing (DMR) is a recent novel endoscopic procedure involving the elective ablation of duodenal mucosa with hydrothermal energy followed by subsequent regeneration, with the aim of treating metabolic disorders [10]. The duodenal mucosa is involved in key metabolic pathways [11] but the underlying molecular mechanisms of this involvement are not fully understood. In a recent multicenter trial that assessed the impact of a single DMR procedure on patients with suboptimally controlled T2DM[12], significant improvement was reported in glycemia, IR, and liver transaminase levels 12 months after the procedure. In addition, noninvasive surrogate markers of liver fibrosis, such as the fibrosis-4 (Fib-4) score, and liver fat changes significantly improved at 6 months and 12 months, respectively, suggesting a potential effect on NAFLD/ NASH [13]. Of note, these outcomes followed modest weight loss and no histopathological liver evaluation was available. Therefore, whether this procedure may also have a positive impact in NASH patients is currently unknown. In this pilot study, we aimed to evaluate the effect of DMR on histologic resolution of NASH and fibrosis improvement in patients with biopsy-confirmed NASH.

\section{Patients and methods}

\section{Study design and patients}

This pilot study was a single-center, single-arm, prospective study that evaluated the effect of a single DMR procedure in patients with biopsy-proven NASH. The study protocol was approved by the Medical Ethics Committee of CUB Hôpital Erasme, Université Libre de Bruxelles, Brussels, Belgium (P2017/302). The study was conducted in accordance with ICH Good Clinical Practice Guidelines and the Declaration of Helsinki. All patients provided written informed consent. Eligible patients were adults (aged $\geq 18$ years) with histological evidence of definite steatohepatitis (per central expert pathologist reading of a liver biopsy obtained $\leq 6$ months prior to the procedure) with an NAFLD activity score (NAS) of at least 4, including at least one point each for steatosis, lobular inflammation, and hepatocellular ballooning; and fibrosis stage, per Brunt stage, of F0 to F3. Main exclusion criteria were as follows: evidence of another cause of liver disease; history of sustained alcohol ingestion defined as daily alcohol consumption $>30 \mathrm{~g} /$ day for males and $>20 \mathrm{~g} /$ day for females; previous gastrointestinal surgery such as Billroth 2, Roux-en-Y gastric bypass, or other similar procedures or conditions; for subjects with T2DM, no current use of insulin or GLP-1 analogs; and for type 1 diabetes, probable insulin production failure defined as fasting $\mathrm{C}$-peptide serum $<1 \mathrm{ng} / \mathrm{mL}$.
The detailed study design, including inclusion and exclusion criteria, can be reviewed in Supplementary TableS1.

\section{Study procedure}

The DMR procedure was performed under general anesthesia by a single senior endoscopist with previous experience in $>20$ cases and patients were hospitalized overnight per protocol. A screening gastroduodenoscopy was conducted first to ensure the absence of a contraindication to the procedure. The DMR device (Revita System; Fractyl Laboratories Inc., Lexington, Massachusetts, United States) (Supplementary Fig. S1) is a single-use catheter that allows submucosal injection and circumferential hydrothermal ablation of the duodenal mucosa, introduced over a guidewire under fluoroscopic visualization, alongside the endoscope, as previously described [12,14]. Endoscopic images of the DMR procedure are presented in Supplementary Fig. S2. Patients were instructed to follow a 2-week diet after DMR in which clear liquids were progressively replaced by solid food. Proton pump inhibitors ( $40 \mathrm{mg}$ once a day) were prescribed for 1 month. General dietary and lifestyle advice were provided before the DMR procedure, according to the European guidelines for the management of NAFLD [15] but no dietary counseling (provided by a dietician) was implemented during the study.

\section{Assessments and outcome measurements}

Follow-up

Follow-up visits were scheduled at 1, 3, 6, and 12 months after DMR in which clinical, anthropometric, biological, endoscopic, radiological, medication use, adverse event $(A E)$, blood pressure, and liver biopsy (at 12 months) assessments were carried out.

\section{Primary endpoint}

The primary endpoint of this proof-of-concept-study was to assess liver histology 12 months after DMR. The primary endpoints were: 1 ) improvement of fibrosis (reduction of at least one stage) with no worsening of NASH (defined as no increase of hepatocellular ballooning, lobular inflammation, or steatosis); and 2) NASH resolution (defined as the overall histopathological interpretation of no fatty liver disease or fatty liver disease without steatohepatitis and an NAS of 0 or $0-1$ for inflammation) with no worsening of fibrosis, as previously defined [18]. The assessment of liver biopsy was performed by an expert independent pathologist who was blinded to patient characteristics at baseline and 12 months after DMR.

\section{Secondary endpoints}

First, we evaluated several markers of liver damage. The levels of alanine aminotransferase (ALT) and aspartate aminotransferase (AST) were determined at each visit. Surrogate markers of liver fibrosis, including Fib-4 and NAFLD fibrosis score, were calculated at baseline and 6 and 12 months after DMR, as previously described [16], using the following formulas: [Age (years) $\times$ AST $(\mathrm{IU} / \mathrm{L})] /[$ plateletcount $\quad(\times 109 / \mathrm{L}) \times \mathrm{ALT}(\mathrm{IU} / \mathrm{L}) 1 / 2]$, for Fib-4, -1.675 + 0.037 - age (years) + $0.094-$ BMI $\left(\mathrm{kg} / \mathrm{m}^{2}\right)+$ 
$1.13 \times \mathrm{IFG} /$ diabetes (yes $=1, \mathrm{no}=0)+0.99 \times \mathrm{AST} / \mathrm{ALT}$ ratio $0.013 \times$ platelet count $\left(\times 10^{9} / \mathrm{l}\right)-0.66 \times$ albumin $(\mathrm{g} / \mathrm{dL})$, for NAFLD fibrosis score.

Magnetic resonance imaging (MRI) (clinical 1.5 Tesla scanner, Achieva, Phillips) was performed at baseline and 6 and 12 months to measure the liver proton density fat fraction (PDFF). All examinations were done by a single senior radiologist blinded to patient characteristics and colocalized regions of interest (ROIs) were used to assess longitudinal fat changes over time. One colocalized ROI was placed in each of nine liver segments and the mean of each ROI was calculated, as previously described [17].

Noninvasive liver stiffness was assessed using vibration-controlled transient elastography (VCTE) (Fibroscan, Echosens, Paris, France) for which 10 valid measurements and an interquartile range to median ratio $<30 \%$ were considered to be reliable [16].

Second, we assessed several metabolic variables. At each follow-up visit, fasting laboratory assessments (i. e. fasting plasma glucose [FPG], glycated hemoglobin [HbA1c], insulin, and Cpeptide) were performed and insulin resistance was estimated by the homeostatic model assessment index for insulin resistance (HOMA-IR), using the following formula: glucose $(\mathrm{mg} / \mathrm{dL}$ ) $x$ insulin/405. A 75-g oral glucose tolerance test (OGTT) (Glucomedics, International Medical products, Belgium) was conducted at each follow-up visit, after a 12-hour overnight fast to evaluate whole-body glucose tolerance [18].

\section{Histopathological evaluation}

Liver and duodenal biopsies were fixed with $4 \%$ formaldehyde and paraffin-embedded. Formalin-fixed paraffin sections were cut at $4 \mu \mathrm{m}$ for liver and duodenal tissues. Liver tissue sections were stained with hematoxylin and eosin (H\&E) and Masson's trichrome. A blinded expert pathologist performed histological grading of the liver tissue sections using the NASH-CRN scoring system. Formalin-fixed duodenal sections were stained with H\&E. Histology images were obtained using a NanoZoomer HT Scan system (Hamamatsu Photonics, Japan).

\section{Adverse events}

Adverse events (AEs) and device-related AEs were recorded at each follow-up visit. Three months after DMR, a gastroduodenoscopy was performed to assess the eventual occurrence of any device-related AEs. Duodenal biopsies were collected before and 3 months after the DMR procedure (Supplementary Fig. S2).

\section{Statistical analysis}

Results for continuous variables are presented as medians (interquartile range) and categorical variables are summarized as frequencies and proportions. The Wilcoxon paired signed-rank test was used to analyze secondary endpoints. For OGTT, a linear mixed model test was used to compare plasma levels of glucose. Corrections for the multiple comparison tests made between time points. The Wilcoxon paired signed-rank test was used to compare two data points during the course of the OGTT. Data are expressed as median (IQR).
Statistical analyses were performed using R statistical analysis software (version 3.6.2, https://www.r-project.org/). All the statistical tests used were two-tailed, and $P<0.05$ was considered statistically significant.

\section{Results}

\section{Patients}

Between 2017 and 2019, fourteen patients with biopsy-confirmed NASH were included in this study, two patients were excluded due to pregnancy and heavy alcohol consumption, respectively, and one patient was lost to follow-up (Supplementary Fig. S3). - Table 1 shows baseline and demographic characteristics for included patients $(n=11)$.

- Table 1 Demographic and baseline clinical characteristics $(N=11)$.

\section{Parameter}

Age, years

$50(47-60)$

Male, $\mathrm{n}(\%)$

$2(18)$

BMI kg/m²

$32.1(28.6-34.8)$

FPG, $\mathrm{mg} / \mathrm{dL}$

116 (95-141)

$\operatorname{HbA} 1 \mathrm{C}(\%)$

$6.5(6.4-6.8)$

HOMA

$6.3(4.5-8.2)$

ALT, U/L

54 (39-90)

AST, U/L

$44(28-60)$

CAP, dB/m

332 (304-344)

Elastometry, $\mathrm{kPa}$

$9.9(8.3-14.1)$

Fib-4

$1.36(1.06-1.54)$

NAFLD fibrosis score

$-1.14(-1.89-0.32)$

Cholesterol, mg/dL

194 (174-214)

Triglycerides, mg/dL

$132(120-158)$

$\mathrm{HDL}, \mathrm{mg} / \mathrm{dL}$

$46(42-54)$

Patients with T2DM, n (\%)

$9(82)$

Fibrosis stage, $n$ (\%)

\begin{tabular}{l|l}
.0 & $2(18)$ \\
\hline .1 & $2(18)$ \\
\hline .2 & $4(36)$ \\
\hline-3 & $2(18)$ \\
\hline .4 & $1(9.1)$ \\
\hline
\end{tabular}

Data are median (IQR) unless otherwise indicated. ALT, alanine aminotransferase; AST, aspartate aminotransferase; BMI, body mass index; CAP, controlled attenuation parameter; Fib-4, fibrosis-4 index; FPG, fasting plasma glucose; HbA1c: glycated hemoglobin; HDL, high density lipoprotein; HOMA: homeostatic model assessment of insulin resistance; NAFLD, nonalcoholic fatty liver disease fibrosis score; T2DM, type 2 diabetes mellitus. 


\section{Primary outcome}

The primary endpoint of NASH resolution with no worsening of fibrosis was not met by any of the patients. The improvement in fibrosis (by at least one stage) with no worsening of NASH was met by three patients (27\%) ( $\vee$ Table 2 ). Among these three patients, two exhibited weight loss of $3 \mathrm{~kg}$ and $4 \mathrm{~kg}$ at 12 months. Based on these results, a theoretical sample size calculation was made to prove DMR efficacy in NASH patients (Supplementary data, Appendix 1).

\section{Secondary outcomes}

The other histological endpoints, including no worsening of fibrosis and $\mathrm{NASH}$, improvement in NAS $\geq 2$ with no worsening of fibrosis, improvement in fibrosis and resolution of NASH as a composite endpoint, improvement in fibrosis by $\geq 2$ stages were not observed in any of the patients. However, improvement of at least one point in steatosis, lobular inflammation, and hepatocellular ballooning were detected in two (18\%), four (36\%), and three patients (27\%) patients, respectively ( Table 2).

The median ALT level (IQR) decreased significantly from 54 $\mathrm{U} / \mathrm{L}(39-90)$ to $44 \mathrm{U} / \mathrm{L}$ at 1 month $(P=0.023)$, but increased to levels of $46 \mathrm{U} / \mathrm{L}(31-74, P=0.21), 47 \mathrm{U} / \mathrm{L}(36-71, P=0.17)$, and $63 \mathrm{U} / \mathrm{L}(30-70, P=0.24)$ at 3,6 , and 12 months, respectively ( $\triangleright$ Fig. 1). The median AST level (IQR) tended to decrease from $44 \mathrm{U} / \mathrm{L}(28-60)$ to $31 \mathrm{U} / \mathrm{L}(25-54, P=0.083)$ at 1 month but remained unchanged from baseline at $3(41 \mathrm{U} / \mathrm{L}(26-50, P=0.33)$, 6 (36U/L (26-48, $P=0.14)$, and 12 months (50U/L (27-63, $P=$ 0.84 ), respectively ( $>$ Fig. 1 ). At 12 months, the number of patients with an ALT level inferior to the upper limit of normal (ULN) (i. e. $40 \mathrm{U} / \mathrm{L}$ ) was 36 (36\%) compared to 27 (27\%) at base-
- Table 2 Efficacy endpoints.

\begin{tabular}{|l|l|}
\hline Primary endpoint, $\mathbf{n}(\%)$ & $3(27)$ \\
\hline \begin{tabular}{l} 
Improvement of fibrosis with no worsening of NASH \\
\hline Resolution of NASH with no worsening of fibrosis
\end{tabular} & 0 \\
\hline Secondary endpoints, $\mathrm{n}(\%)$ & $3(27)$ \\
\hline $\begin{array}{l}\text { Improvement of fibrosis by } \geq 1 \text { stage or resolution of NASH } \\
\text { without worsening of either }\end{array}$ & 0 \\
\hline \begin{tabular}{l} 
No worsening of fibrosis and no worsening of NASH \\
\hline Improvement of NAS by $\geq 2$ with no worsening of fibrosis
\end{tabular} & 0 \\
\hline $\begin{array}{l}\text { Improvement of fibrosis and resolution of NASH as a com- } \\
\text { posite endpoint }\end{array}$ & 0 \\
\hline \begin{tabular}{l} 
Improvement in fibrosis by $\geq 2$ stages \\
\hline Resolution of fibrosis
\end{tabular} & 0 \\
\hline$\geq 1$ point improvement in steatosis & 0 \\
\hline$\geq 1$ point improvement in lobular inflammation & $4(36)$ \\
\hline$\geq 1$ point improvement in hepatocellular ballooning & $3(27)$ \\
\hline $\begin{array}{l}\text { NAS, nonalcoholic fatty liver disease activity score; NASH, nonalcoholic } \\
\text { steatohepatitis. }\end{array}$
\end{tabular}

line, whereas an AST level inferior to the ULN was seen in 36 (36\%) at 12 months compared to 45 (45\%) at baseline.

After DMR, the median Fib-4 score (IQR) slightly improved from $1.36(1.06-1.54)$ to $1.20(0.98-1.34)$ and $1.26(1.09-$ $1.90)$ at 6 and 12 months, respectively, but the median change was not significantly different ( $\triangleright$ Fig. 2). Similarly, there was no improvement in median NAFLD fibrosis score (IQR) which in-

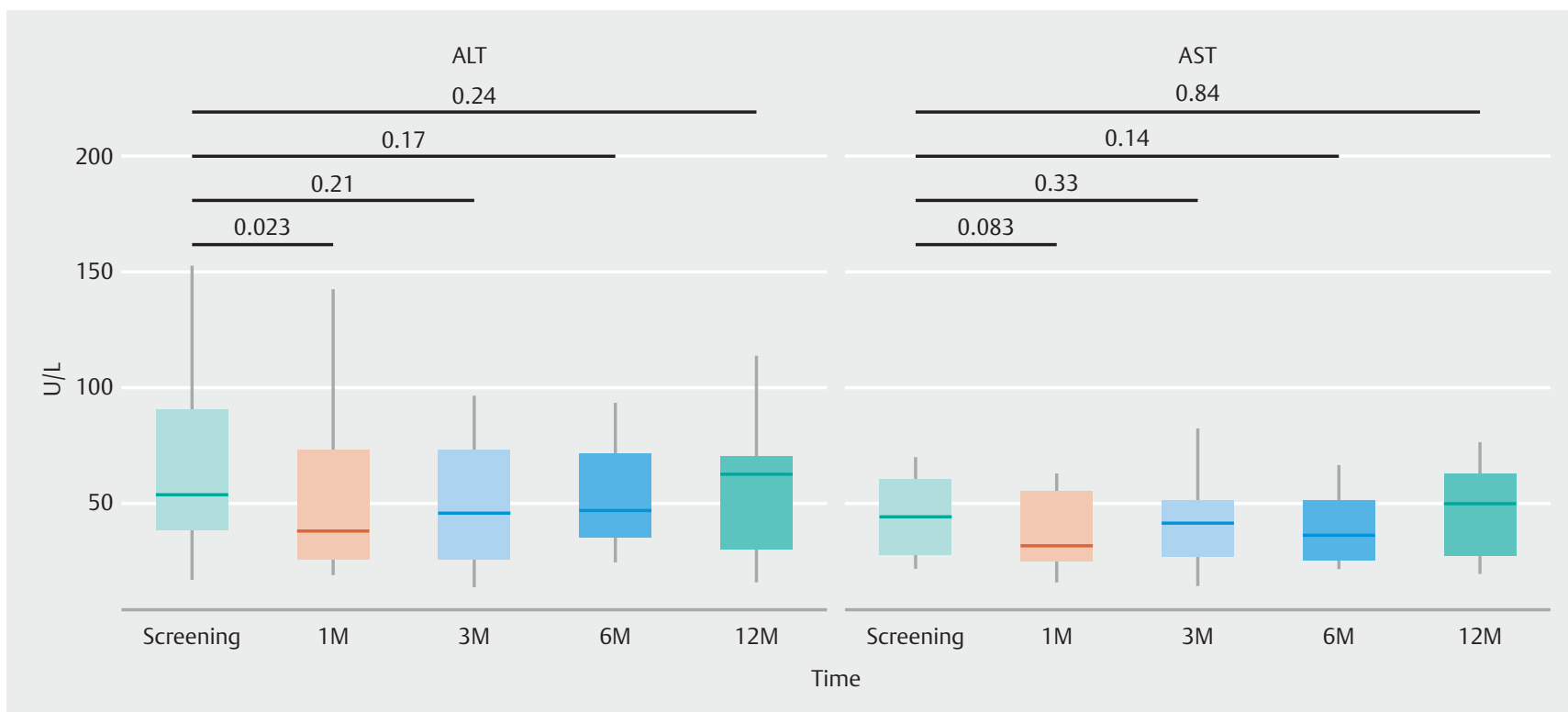

- Fig. 1 Change in liver transaminase levels over time. Change in liver biochemistry over time. Box plots of alanine aminotransferase (ALT) and aspartate aminotransferase (AST) at 1 month (1 M), 3 months ( $3 \mathrm{M}) ; 6$ months (6 M), and 12 months (12M). Boxes indicate the 25th to 75th percentiles, the line within the boxes marks the median, whiskers (error bars) above and below the box indicate the 90th and 10th percentiles, while $P$ values are indicated within the graph. 


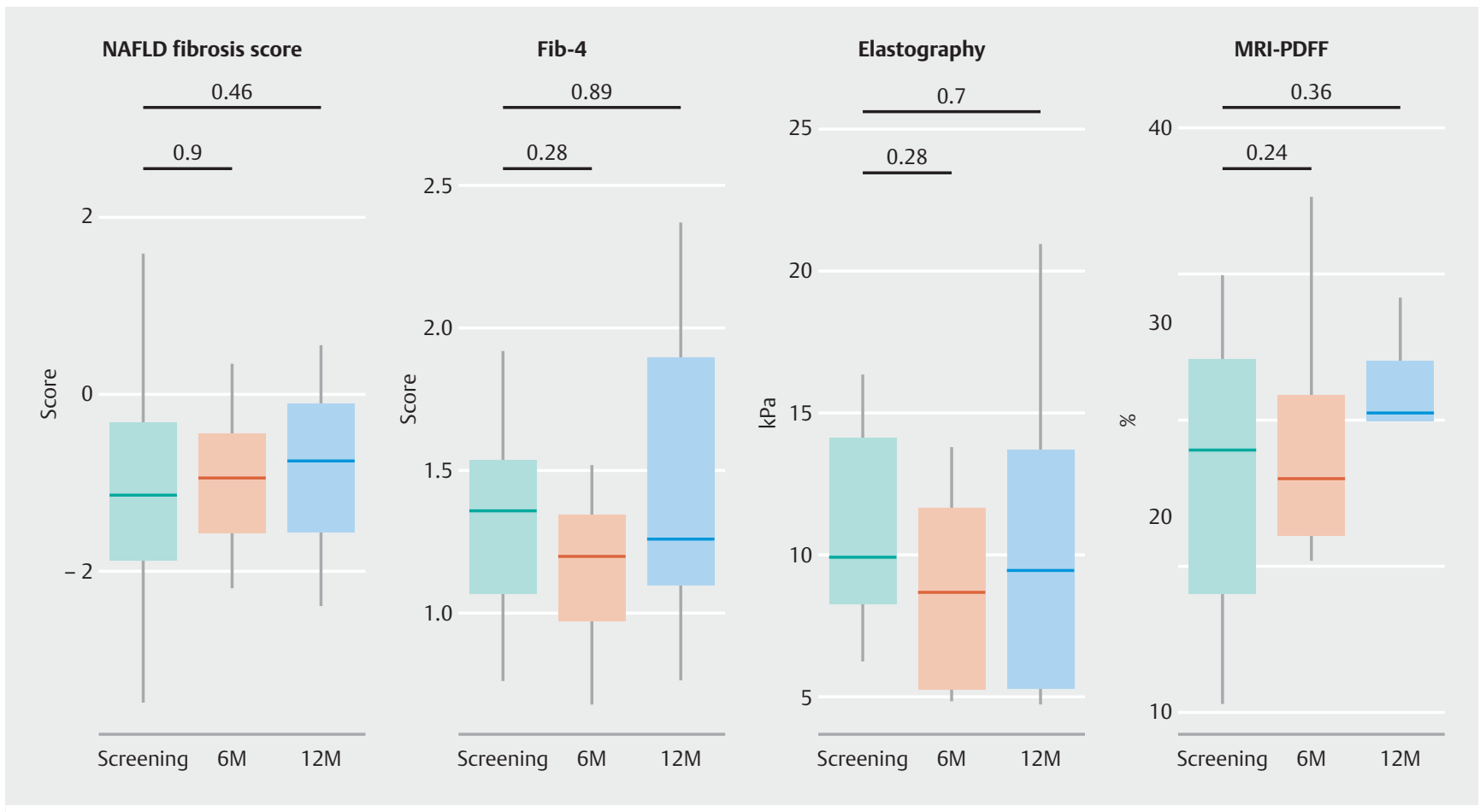

Fig. 2 Change in surrogate markers of liver fibrosis and steatosis over time. Change in surrogate markers of liver fibrosis and steatosis over time. Box plots of nonalcoholic fatty liver disease fibrosis score (NAFLD), fibrosis-4 score (Fib-4), elastometry, and magnetic resonance imaging-derived proton density fat fraction (MRI-PDFF) at 6 months ( $6 \mathrm{M})$ and 12 months (12 M). Boxes indicate the 25th to 75th percentiles, the line within the boxes marks the median, whiskers (error bars) above and below the box indicate the 90th and 10th percentiles, while $P$ values are indicated within the graph.

creased from $-1.14(-1.89--0.32)$ to $-0.95(-1.58--0.46, P=$ $0.9)$ and $-0.75(-1.56--0.10, P=0.46)$ at 6 and 12 months, respectively ( $\mathbf{F i g . 2}$ ). The median VCTE (IQR) also did not change significantly from $9.8 \mathrm{kPa}(8.2-14.1)$ to $8.6 \mathrm{kPa}(5.2-$ 11.6, $P=0.28)$ and $9.4 \mathrm{kPa}(5.3-13.7, P=0.7)$ at 6 and 12 months, respectively ( Fig. 2 ). The median MRI-PDFF (IQR) values did not reveal any change from $23 \%(16-28)$ at baseline to $22 \%(19-26, P=0.24)$ at 6 months and $25 \%(25-28, P=0.46) 12$ months after DMR, respectively ( $\triangleright$ Fig. 2 ). The median glycemic parameters derived from OGTT were statistically significant different $(P=0.06)$ compared to baseline values at 1 month ( $\triangleright$ Fig. 3a). The median plasma glucose level (IQR) decreased significantly from $230 \mathrm{mg} / \mathrm{dL}(210.5-307)$ to $199 \mathrm{mg} / \mathrm{dL}$ (161.5-277) 60 minutes after glucose load $(P<0.05)$ and from $231 \mathrm{mg} / \mathrm{dL}$ (206.5-315.5) to $214 \mathrm{mg} / \mathrm{dL}(177-297) 90$ minutes after glucose load $(P<0.05)$.

Median weight (IQR) was similar at 1, 6, and 12 months after DMR while a significant weight loss was observed at 3-month follow-up (from $82 \mathrm{~kg}$ [76-94] to $80 \mathrm{~kg}$ [76-89]; $P=0.0083$ ) ( Fig.3b).

The median HbA1c level (IQR) significantly decreased 1 month after DMR, from $6.50 \%(6.35-6.75)$ to $6.20 \%(6.10-$ $6.40, P=0.014)$ and was sustained at 3 and 6 months, although the median change was not statistically different at these time points. This improvement was not sustained at 12 months where the median HbA1c level increased to 6.60 (6.15-6.75, $P$ $=0.68)$ ( $\triangleright$ Fig. 3c).
The median HOMA index (IQR) significantly decreased 1 month after DMR, from $6.3(4.5-8.2)$ to $5.2(3.2-6.8, P=$ 0.019). Surprisingly, the median HOMA level increased at 3,6 , and 12 months compared to baseline value to $7.2(5.5-8.4, P=$ $0.83)$, $8.3(4.5-11.1, P=0.58)$, and 6.8 (5.9-10.6, $P=0.46)$, respectively ( $\triangleright$ Fig. $\mathbf{3 d}$ ).

\section{Characteristics of DMR procedure and adverse events}

The median DMR procedure time (IQR) was 62 minutes (54.5$68)$ whereas the median number of successive duodenal mucosal hydrothermal balloon ablations (IQR) was seven (6-8). All patients underwent a gastroscopy 3 months after DMR, with no findings of duodenal stenosis or gastroduodenal ulcers in any of the patients.

Overall, two serious AEs (SAEs) (14\%) related to the procedure occurred in this pilot study. One patient presented with moderate hypoxemia due to atelectasis of the right lower lobe of the lung and was successfully treated with oxygen and physiotherapy. The patient was discharged 2 days after the procedure. The second patient presented with epigastric pain associated with mild fever and moderate elevation in C-reactive protein. No microbiologically documented infections or local duodenal complications due to DMR were found on computed tomography scan. The evolution was spontaneously favorable and the patient was discharged 4 days after the procedure. Despite the absence of clear evidence, we cannot exclude that this latter event could be potentially related to the DMR procedure. 


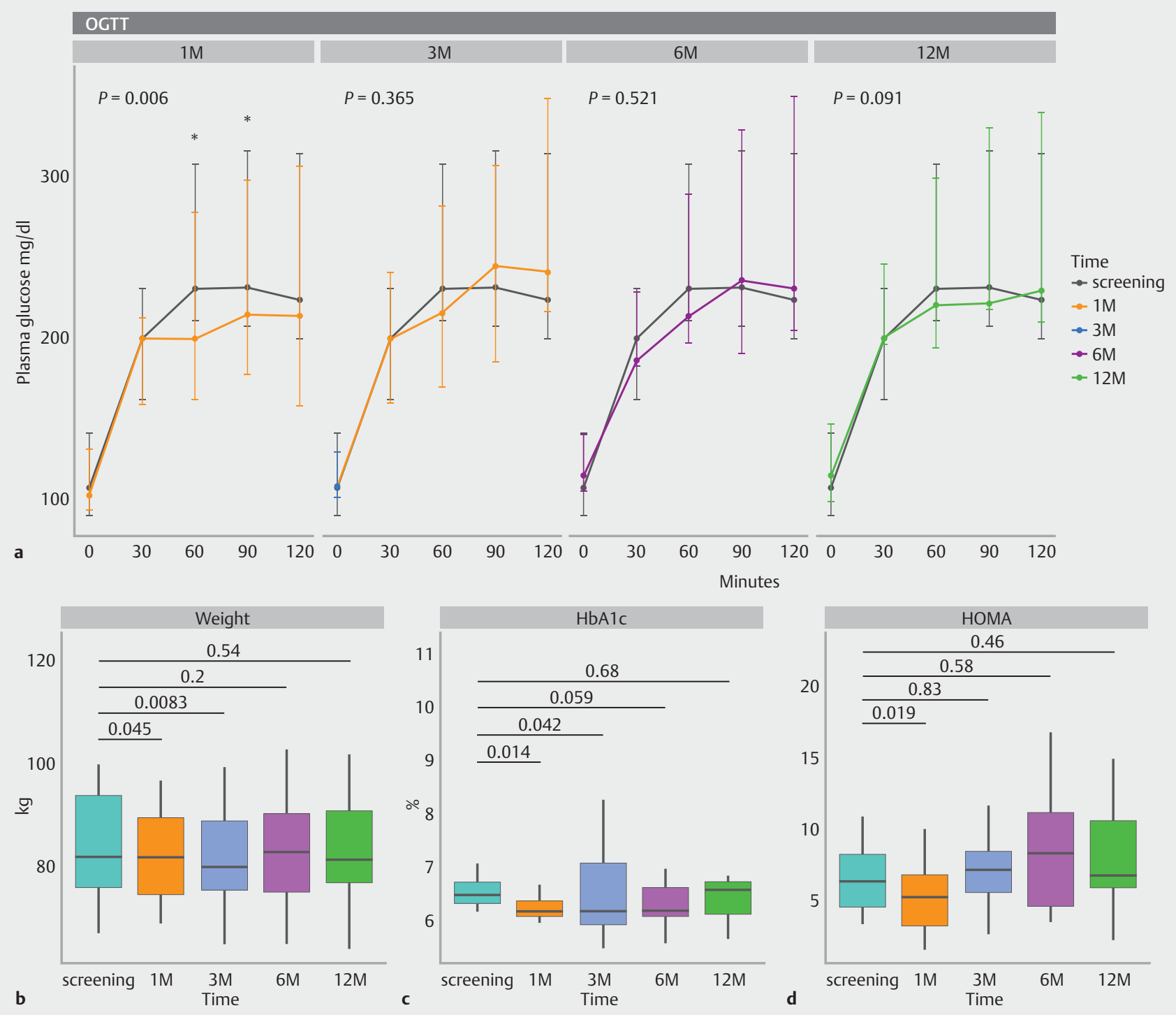

- Fig. 3 Change in metabolic parameters over time. Change in metabolic parameters over time. a Oral glucose tolerance test (OGTT), plasma glucose at baseline and 30,60, 90, 120 minutes (Min) after ingestion of $75 \mathrm{~g}$ of oral glucose. Data are expressed as median (IQR). Significance $(P<0.05)$ between curves was assessed by mixed model test ( $\mathrm{p}$ values indicated within the graph). ${ }^{*}$ Indicates a $P<0.05 . N=11$, nine of whom had type 2 diabetes. b, c, $\mathbf{d}$ Box plots of weight, glycated hemoglobin (HbA1c), and homeostatic model of insulin resistance (HOMA) at screening at 1 month $(1 \mathrm{M}), 3$ months $(3 \mathrm{M}), 6$ months $(6 \mathrm{M})$, and 12 months $(12 \mathrm{M})$. Boxes indicate the 25 th to 75 th percentiles, the line within the boxes marks the median, whiskers (error bars) above and below the box indicate the 90 th and 10 th percentiles, while $P$ values are indicated within the graph.

No unanticipated adverse device events (UADEs) were reported.

The most frequent AEs ( $\triangleright$ Table 3 ) were related to gastrointestinal disorders (36\%) and comprised the following symptoms: heartburn, constipation, chest wall pain, and epigastric pain. Two patients experienced hyperglycemia, one was mild and related to discontinuation of antidiabetic drugs and was resolved after resuming appropriate medication whereas the other patient needed to tailor their therapy by adding a second antidiabetic drug (i.e., gliclazide). Of note, this patient presented with decompensated diabetes, the latter being difficult to adapt despite tailoring antidiabetic drugs. The changes in oral antidiabetic medication are presented in Supplementary Table S2.

\section{Discussion}

This pilot study assessed, for the first time, the DMR procedure in patients with biopsy-confirmed NASH with histological evaluation at 1 year. Aside from a marginal improvement in fibrosis score, we found no resolution of NASH and no improvement in other liver histology or metabolic markers 12 months after the procedure. Similarly, MRI, PDFF, and VCTE also remained unchanged at 1 year. Of note, no intensive lifestyle follow-up was 
- Table 3 Summary of adverse events.

\begin{tabular}{|c|c|}
\hline Adverse events & $\begin{array}{l}\text { Number of } \\
\text { patients (\%) } \\
\mathrm{N}=14\end{array}$ \\
\hline $\begin{array}{l}\text { Serious adverse events possibly or related to the } \\
\text { procedure }\end{array}$ & $2(14)$ \\
\hline \multicolumn{2}{|c|}{$\begin{array}{l}\text { Gastrointestinal disorders related to the procedure including heart- } \\
\text { burn, constipation, chest wall pain, epigastric pain }\end{array}$} \\
\hline - Mild & $5(36)$ \\
\hline - Moderate & 0 \\
\hline - Severe & $1(7)$ \\
\hline \multicolumn{2}{|c|}{$\begin{array}{l}\text { Endocrine disorders not related to the procedure including hypergly- } \\
\text { cemia }\end{array}$} \\
\hline - Mild & $1(7)$ \\
\hline - Moderate & $1(7)$ \\
\hline - Severe & 0 \\
\hline \multicolumn{2}{|c|}{$\begin{array}{l}\text { Musculoskeletal and connective tissue disorders not related to the } \\
\text { procedure }\end{array}$} \\
\hline - Mild & $1(7)$ \\
\hline - Moderate & 0 \\
\hline - Severe & 0 \\
\hline \multicolumn{2}{|c|}{$\begin{array}{l}\text { Nervous system disorders not related to the procedure including falls } \\
\text { without loss of consciousness }\end{array}$} \\
\hline - Mild & $1(7)$ \\
\hline - Moderate & 0 \\
\hline - Severe & 0 \\
\hline \multicolumn{2}{|c|}{$\begin{array}{l}\text { Respiratory, thoracic and mediastinal disorders related to the proce- } \\
\text { dure including hypoxemia }\end{array}$} \\
\hline - Mild & 0 \\
\hline - Moderate & 0 \\
\hline - Severe & $1(7)$ \\
\hline
\end{tabular}

proposed during the study and patient weights remained unchanged at 1 year.

Our study population differs from the recent published REVITA-2 randomized clinical trial (RCT) [19] in which DMR was shown to be associated with improvement in metabolic factors. First, a greater reduction in $\mathrm{HbA} 1 \mathrm{c}$ was reported in patients with high baseline FPG $(\geq 180 \mathrm{mg} / \mathrm{d})$ which contrasts with the demographic data for our study population. In addition, our study was designed to avoid the potential bias of weight loss because no intensive dietary intervention was implemented over the course of the trial and, as expected, no significant weight loss was observed 12 months after DMR. More specifically, comprehensive comparison of our study with REVITA-2 and the INSPIRE [20] studies is difficult because not only outcomes were evaluated at different timepoints, but mostly, especially for the INSPIRE study, the design was different em- phasized by the implementation of another drug, i.e. glucagon-like peptide-1 receptor agonist. Liver fat content (measured by PDFF) was assessed at 3 months in the REVITA-2 study whereas long-term data (i.e. 12 months) were available in the INSPIRE study. Interestingly, the median baseline PDFF value was of $8.1 \%(4.0-13.5)$ while our study reported a higher baseline value of $23 \%$ (16-28). Moreover, liver fat content improvement reported in the INSPIRE study was associated with a significant weight loss whereas the level of weight change was not detailed at 3 months in the REVITA- 2 study. These findings underscore that our study population is more severe compared to the published data and NAFLD is commonly known to be heterogenous [21]. In the other hand, weight loss achieved with a hypocaloric diet and/or increased physical activity is known to improve NASH-related histological endpoints [6,15,22] and metabolic factors such as HbA1c and HOMA-IR [23]. Controlling this important confounder was, therefore, critical to identifying a potential impact of DMR in NASH patients. Accordingly, most clinical trials in NASH have not provided nutritional counseling or an accurate description of lifestyle modifications [24]. Importantly, in a recent meta-analysis of placebo groups from 39 biopsy-proven NASH RCTs, $25 \%$ of patients in the placebo groups had an improvement in NAS of $\geq 2$ points and $21 \%$ had an improved fibrosis score and these changes were correlated to changes in body mass index [25]. In this study, three patients ( $27 \%$ ) had an improved fibrosis score (by one stage) with no worsening of NASH; however, this might, at least in part, reflect the impact of weight loss observed in two of them. Thus, this apparently positive histological effect might be in line with the placebo response observed in large multicentric NASH clinical trials [25].

Furthermore, our pilot study population consisted of biopsyconfirmed NASH patients, more than $25 \%$ of whom had advanced fibrosis, a feature that may also explain the discrepancies between our results and those reported in previous DMR trials performed in T2DM patients [12,13]. Even though T2DM and NAFLD often co-exist [4], these findings support the fact that our population represents a more severe group, probably related to the selection of patients with biopsy-proven NASH. This could explain, at least in part, the negative results observed in this study.

No UADEs occurred in this pilot study and, more specifically, no duodenal stenosis, a factor that was a concern with the first published DMR trial [26]. Since then, further improvements were made to ensure proper mucosal lifting before ablation to avoid the aforementioned complications and a second-generation catheter was used in our study. The two SAEs related to the procedure (i.e. mild hypoxemia after extubation and transient fever) were not related to the device and resolved without further intervention within 1 to 3 days.

This pilot study has some limitations. The sample size was limited in the setting of a highly heterogeneous disease. However, the study was initially designed to evaluate, in a specific population of patients with biopsy-proven NASH whether DMR could have a positive effect on liver histology endpoints, the gold standard to prove a therapeutic role. The purpose of this pilot study was to evaluate whether at least a trend toward a 
positive effect could be detected. This would have led to the conduct of a larger multicenter study to prove DMR efficacy in NASH patients, a project obviously abandoned given the current results. Other limitations are related to the observational design of the study and the high dropout rate. This study was also not designed to investigate the pathophysiological role of the duodenal mucosa. Further studies are required to better understand the underlying mechanisms induced by DMR and the possible synergistic effect of weight loss. Such explorations might be difficult to assess in humans, and in vitro and/or rodent models could be very helpful in this setting.

\section{Conclusions}

In conclusion, in this small and heterogenous study population, the DMR procedure in patients with biopsy-proven NASH did not improve liver histological endpoints, liver fat content, or any other metabolic marker at 12 months, in the absence of weight loss.

\section{Acknowledgements}

The authors thank all study participants, research study nurses, and nurses involved in DMR procedures. We would like to acknowledge the contribution of a medical writer, Sandy Field, $\mathrm{PhD}$, for English language editing of this manuscript.

\section{Competing interests}

This investigator-initiated study was supported in part through a grant from Fractyl Inc., Lexington, Massachusetts, United States. Dr. Devière receives institutional research support for IRB-approved studies from Fractyl Inc, Lexington, Massachusetts, United States. Dr. Hadefi is supported by a research grant from the "Fonds Erasme pour la recherche médicale" (doctoral research fellow grant). Dr. Trépo is a Research Associate of the Fund for Scientific Research-FNRS.

\section{References}

[1] Younossi ZM, Golabi P, de Avila L et al. The global epidemiology of NAFLD and NASH in patients with type 2 diabetes: A systematic review and meta-analysis. J Hepatol 2019; 71: 793-801

[2] Huang DQ, El-Serag HB, Loomba R. Global epidemiology of NAFLDrelated HCC: trends, predictions, risk factors and prevention. Nat Rev Gastroenterol Hepatol 2020: doi:10.1038/s41575-020-00381-6 Epub 2020 Dec 21

[3] Younossi ZM. Long-term outcomes of nonalcoholic fatty liver disease: from nonalcoholic steatohepatitis to nonalcoholic steatofibrosis. Clin Gastroenterol Hepatol 2017; 15: 1144-1147

[4] Eslam M, Newsome PN, Sarin SK et al. A new definition for metabolic dysfunction-associated fatty liver disease: An international expert consensus statement. J Hepatol 2020; 73: 202-209

[5] Davies MJ, D’Alessio DA, Fradkin J et al. Management of Hyperglycemia in Type 2 Diabetes, 2018. A Consensus Report by the American Diabetes Association (ADA) and the European Association for the Study of Diabetes (EASD). Diabetes Care 2018; 41: 2669-2701

[6] Vilar-Gomez E, Martinez-Perez Y, Calzadilla-Bertot L et al. Weight loss through lifestyle modification significantly reduces features of nonalcoholic steatohepatitis. Gastroenterology 2015; 149: 367-378.e5
[7] Koutoukidis DA, Astbury NM, Tudor KE et al. Association of weight loss interventions with changes in biomarkers of nonalcoholic fatty liver disease: a systematic review and meta-analysis. JAMA Intern Med 2019; 179: 1262

[8] Romero-Gómez M, Zelber-Sagi S, Trenell M. Treatment of NAFLD with diet, physical activity and exercise. J Hepatol 2017; 67: 829-846

[9] Lassailly G, Caiazzo R, Ntandja-Wandji LC et al. Bariatric surgery provides long-term resolution of nonalcoholic steatohepatitis and regression of fibrosis. Gastroenterology 2020; 159: 1290-1301.e5

[10] Rajagopalan H, Cherrington AD, Thompson CC et al. Endoscopic duodenal mucosal resurfacing for the treatment of type 2 diabetes: 6-month interim analysis from the first-in-human proof-of-concept study. Diabetes Care 2016; 39: 2254-2261

[11] Abu Dayyeh BK, Bazerbachi F, Graupera I et al. Endoscopic bariatric and metabolic therapies for non-alcoholic fatty liver disease. J Hepatol 2019; 71: 1246-1248

[12] Van Baar ACG, Holleman F, Crenier L et al. Endoscopic duodenal mucosal resurfacing for the treatment of type 2 diabetes mellitus: One year results from the first international, open-label, prospective, multicentre study. Gut 2020; 69: 295-303

[13] van Baar ACG, Beuers U, Wong K et al. Endoscopic duodenal mucosal resurfacing improves glycaemic and hepatic indices in type 2 diabetes: 6-month multicentre results. JHEP Reports 2019; 1: 429-437

[14] Haidry R], van Baar AC, Galvao Neto MP et al. Duodenal mucosal resurfacing: proof-of-concept, procedural development, and initial implementation in the clinical setting. Gastrointest Endosc 2019; 90: 673-681.e2

[15] European Association for the Study of the Liver (EASL), European Association for the Study of Diabetes (EASD), European Association for the Study of Obesity (EASO). EASL-EASD-EASO Clinical Practice Guidelines for the management of non-alcoholic fatty liver disease. J Hepatol 2016; 64: 1388-1402

[16] Castera L, Friedrich-Rust M, Loomba R. Noninvasive Assessment of liver disease in patients with nonalcoholic fatty liver disease. Gastroenterology 2019; 156: 1264-1281.e4

[17] Cui ], Philo L, Nguyen P et al. Sitagliptin vs. placebo for non-alcoholic fatty liver disease: A randomized controlled trial. . J Hepatol 2016; 65: $369-376$

[18] Matsuda M, DeFronzo RA. Insulin sensitivity indices obtained from oral glucose tolerance testing: Comparison with the euglycemic insulin clamp. Diabetes Care 1999; 22: 1462-1470

[19] Mingrone G, van Baar AC, Devière J et al. Safety and efficacy of hydrothermal duodenal mucosal resurfacing in patients with type 2 diabetes: the randomised, double-blind, sham-controlled, multicentre REVITA-2 feasibility trial. Gut 2021: doi:10.1136/gutjnl-2020323608

[20] van Baar ACG, Meiring S, Smeele P et al. Duodenal mucosal resurfacing combined with glucagon-like peptide-1 receptor agonism to discontinue insulin in type 2 diabetes: a feasibility study. Gastrointest Endosc 2021; 94: 111-120.e3

[21] Eslam M, Sanyal AJ, George J et al. MAFLD: A consensus-driven proposed nomenclature for metabolic associated fatty liver disease. Gastroenterology 2020; 158: 1999-2014.e1

[22] Chalasani N, Younossi Z, Lavine JE et al. The diagnosis and management of nonalcoholic fatty liver disease: Practice guidance from the American Association for the Study of Liver Diseases. Hepatology 2018; 67: 328-357

[23] Look AHEAD Research Group. Cardiovascular Effects of Intensive Lifestyle Intervention in Type 2 Diabetes. N Engl J Med 2013; 369: 145-154

[24] Glass O, Filozof C, Noureddin M et al. Standardisation of diet and exercise in clinical trials of NAFLD-NASH: Recommendations from the Liver Forum. J Hepatol 2020; 73: 680-693 
[25] Han MAT, Altayar O, Hamdeh S et al. Rates of and factors associated with placebo response in trials of pharmacotherapies for nonalcoholic steatohepatitis: systematic review and meta-analysis. Clin Gastroenterol Hepatol 2019; 17: 616-629.e26
[26] Rajagopalan H, Cherrington AD, Thompson CC et al. Endoscopic duodenal mucosal resurfacing for the treatment of type 2 diabetes: 6-month interim analysis from the first-in-human proof-of-concept study. Diabetes Care 2016; 39: 2254-2261 